\title{
Modelo conceptual para diseñar un plan curricular auxiliar sostenible en periodos de contingencia para las instituciones públicas de educación superior
}

\author{
Conceptual model to design a sustainable auxiliary curriculum plan in \\ contingency periods for public institutions of higher education
}

\section{Modelo conceitual para elaboração de plano curricular auxiliar sustentável em períodos de contingência para instituições públicas de ensino superior}

Alicia Navarrete Hernández

Instituto Politécnico Nacional, Escuela Superior de Turismo, México

mixta.est.ipn@gmail.mx https://orcid.org/0000-0002-3803-3946

\section{Resumen}

El presente trabajo es el resultado del estudio de una herramienta innovadora de diseño curricular para la educación del desarrollo sostenible. El principal objetivo fue crear un instrumento estratégico de gestión para situaciones críticas durante el proceso de enseñanza y aprendizaje. Se analizó el método de diseño curricular de tres instituciones públicas de educación superior, tres postulados internacionales para la educación del desarrollo sostenible, dos teorías de aprendizaje constructivista basado en el andamiaje, una teoría para el diseño de estrategias de aprendizaje, así como referencias acerca de los estilos y modelos de aprendizaje.

El estudio se basó en los métodos analítico, sistémico e inductivo, a través de los cuales se obtuvieron datos acerca del contexto de las principales teorías de aprendizaje constructivista, métodos de diseño curricular, postulados acerca de la educación para el desarrollo sostenible, tendencias tecnológicas en la educación, tipos y modelos de aprendizaje, y formas de enseñanza. 


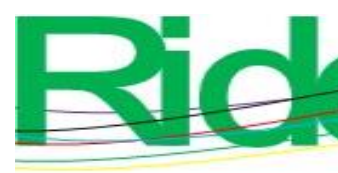
Revista Iberoamericana para la
Investigación y el Desarrollo Educativo
ISSN $2007-7467$

it was identified that the method is diverse with the exception of the curricular model since in two institutions it is systemic and in one it is modular.

The topics related to the 2030 agenda for sustainable development, in the curricular content of study programs, favor the quality of education. It is suggested to integrate into the curriculum design method a sustainable auxiliary plan for the prevention of critical situations and also to incorporate topics related to sustainable development that serve as a complement to the current program content and that promote sustainable meaningful learning to respond to derived critical situations of natural phenomena caused by climate change.

Keywords: education for sustainable development, higher education, educational trend, curriculum, educational innovation.

\section{Resumo}

O presente trabalho é o resultado do estudo de uma ferramenta inovadora de desenho curricular para a educação para o desenvolvimento sustentável. O objetivo principal foi criar um instrumento de gestão estratégica para situações críticas durante o processo de ensino e aprendizagem. O método de concepção curricular de três instituições públicas de ensino superior, três postulados internacionais para a educação para o desenvolvimento sustentável, duas teorias construtivistas de aprendizagem baseadas em andaimes, uma teoria para a concepção de estratégias de aprendizagem, bem como referências sobre estilos e modelos de aprendizagem.

O estudo baseou-se em métodos analíticos, sistémicos e indutivos, através dos quais foram obtidos dados sobre o contexto das principais teorias construtivistas de aprendizagem, métodos de concepção curricular, postulados sobre educação para o desenvolvimento sustentável, tendências tecnológicas em. educação, tipos e modelos de aprendizagem e formas de ensino.

Observou-se que nenhum dos métodos utilizados pelas três instituições públicas de ensino superior possui plano de contingência para situações críticas dentro da estrutura do método de desenho curricular, embora ações tenham sido implementadas para solucionar os problemas apresentados. Quanto à sua estrutura, identificou-se que o método é diverso, com exceção do modelo curricular, visto que em duas instituições é sistêmico e em uma é modular. 


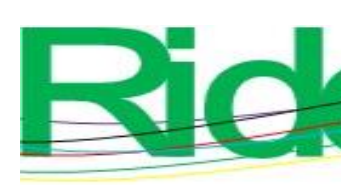

Revista Iberoamericana para la Investigación y el Desarrollo Educativo ISSN $2007-7467$

Os temas relacionados à agenda 2030 para o desenvolvimento sustentável nos conteúdos curriculares dos programas de estudos favorecem a qualidade da educação. Sugere-se integrar no método de desenho curricular um plano auxiliar sustentável para a prevenção de situações críticas e também incorporar questões relacionadas ao desenvolvimento sustentável que complementem o conteúdo programático atual e que promovam uma aprendizagem significativa e sustentável para responder às situações críticas que surgem. de fenômenos naturais causados pelas mudanças climáticas.

Palavras-chave: educação para o desenvolvimento sustentável, ensino superior, inovação educacional, currículo, tendência educacional.

Fecha Recepción: Junio 2020

Fecha Aceptación: Noviembre 2020

\section{Introducción}

El planteamiento teórico y conceptual del desarrollo sostenible surge cuando la Comisión sobre el Medio Ambiente y Desarrollo de las Naciones Unidas presentó en el año 1987 el Informe Brundtland, denominado Nuestro Futuro Común. En el documento se plasma el concepto desarrollo sostenible, el cual se presenta como una alternativa para buscar el progreso con base en el aprovechamiento racional de los recursos naturales y humanos. Por ello, se define como "aquel que satisface las necesidades del presente, sin comprometer las necesidades de las futuras generaciones" (Organización de las Naciones Unidas para la Educación, la Ciencia y la Cultura [Unesco], 2012, p. 5). Este término, en otras palabras, se usa para referirse a la necesidad de hacer grandes cambios no solo en cuanto a la sensibilidad por la naturaleza, sino principalmente en los sistemas nacionales e internacionales de producción y consumo, de ahí que haya servido para promover diversos debates internacionales.

En 2002, por ejemplo, se llevó a cabo la Cumbre Mundial sobre el Desarrollo Sostenible, denominada Cumbre de Johannesburgo, Río+10, en donde se expuso la propuesta para el decenio de las Naciones Unidas de la Educación para el Desarrollo Sostenible, la cual se aprobó hasta el 2005. En 2013 la Unesco — para dar seguimiento a esa iniciativa - presentó el programa de acción mundial de Educación para el Desarrollo Sostenible, y en 2014 — en la Reunión Mundial sobre la Educación para Todos (EPT) — se incorporó un objetivo global para el 2030. Luego, en 2015 se realizó el Foro Mundial sobre la Educación (celebrado en Incheon, República de Corea), donde se efectuaron seis debates 


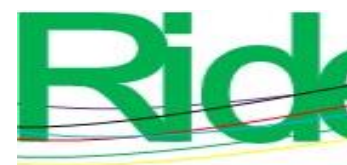
Revista Iberoamericana para la
Investigación y el Desarrollo Educativo
ISSN $2007-7467$

temáticos y se generó un acuerdo para unir a la comunidad educativa en una visión común a través de la agenda educativa 2015-2030 (Unesco, 2017).

Asimismo, también en 2015 se llevó a cabo la Cumbre de las Naciones Unidas sobre el Desarrollo Sostenible, donde ciento noventa y tres estados miembros aprobaron la agenda para el desarrollo sostenible, la cual fue plasmada en el documento denominado Transformar nuestro mundo: la agenda 2030 para el desarrollo sostenible (Organización de las Naciones Unidas [ONU], 2015, p. 1). En este se incluyeron diecisiete objetivos para el desarrollo sostenible, de los cuales el número 4 se enfocó en la educación de calidad para mejorar la vida en el planeta a través del desarrollo sostenible. Con base en esta visión, la situación que se vive actualmente con la pandemia en el mundo adquiere mayor relevancia. Al respecto, la Organización Mundial de la Salud (OMS) ha manifestado que "las medidas urgentes adoptadas por las ciudades y otros asentamientos urbanos en relación con la COVID-19, deben sentar las bases del desarrollo sostenible, de la capacidad para afrontar emergencias de salud simultáneas o futuras" (OMS, 2020, p. 10).

En este contexto, se hacen cada vez más pertinentes estrategias como el aprendizaje híbrido (o b-learning), que se basa en un modelo que surge en el ámbito empresarial, y que se apoya en un pensamiento ecléctico y práctico (Bartolomé y Aiello, 2006). Salinas, De Benito, Pérez y Gisbert (2018) refieren que esta metodología combina estrategias didácticas presenciales con el uso de tecnología para mejorar y reforzar la experiencia de aprendizaje mediante diferentes modelos, como los que se explican a continuación:

- Modelo de rotación: Consiste en rotar la modalidad de aprendizaje a través de diferentes enfoques: aula invertida, aprendizaje en el aula y en línea, en laboratorio $\mathrm{o}$ individual.

- Modelo flexible: Es la combinación de la modalidad presencial y en línea; se utiliza para el desarrollo de proyectos o tutorías.

- Modelo a la carta: En este el estudiante tiene la opción de inscribir un porcentaje de materias en línea y otro presencial.

- Modelo virtual enriquecido: Facilita al estudiante realizar la mayor parte de curso en línea fuera de la institución; además, asiste a clase presencial con un tutor para retroalimentar el aprendizaje. 


\section{Marco de referencia}

Hablar del impacto que tiene la covid-19 en la educación para el desarrollo sostenible significa que los programas internacionales propuestos para impulsar la transformación de la educación para el 2030 requieren de un análisis de causa y efecto acerca del contexto actual generado por la pandemia, ya que de esa manera se puede conocer el punto de partida después del confinamiento, así como las oportunidades para mejorar la educación para el desarrollo sostenible propuestas en 2015.

De acuerdo con las cifras proporcionadas en el mes de marzo de 2020 en el monitoreo del seguimiento mundial de los cierres de escuelas causados por el Covid-19 de la Coalición Mundial para la Educación de la Unesco, el confinamiento "afectó la educación de más de 1500 millones de estudiantes a nivel mundial en 185 países que han cerrado escuelas y universidades en todo el territorio, lo cual afectó al $89.4 \%$ de la población estudiantil en el planeta"(párr. 3). En consecuencia, la Unesco ha promovido acciones para mitigar el impacto, principalmente en las comunidades más vulnerables para facilitar la continuidad de la educación a través del aprendizaje remoto. En el caso de México, se implementó un programa especial para todos los alumnos del nivel básico denominado Aprende en casa por TV. Además, las instituciones educativas públicas y privadas han desarrollado sus propias estrategias de aprendizaje en línea por medio del uso de diversas plataformas, así como de herramientas digitales y electrónicas para fomentar el aprendizaje significativo en los estudiantes.

Por otra parte, el Instituto Nacional de Estadística y Geografía (Inegi) (2019), en la Encuesta Nacional sobre Disponibilidad y Uso de Tecnologías de la Información en los Hogares, ha señalado que en México hay 80.6 millones de usuarios de internet, lo que representa $70.1 \%$ de la población con seis años de edad o más. También se estima que en 20.1 millones de hogares se dispone de internet, lo que constituye 56.4\% del total general. Los principales medios de conexión a internet son los smartphone (95.3\%), la computadora portátil $(33.3 \%)$ y la computadora de escritorio (28.9\%). La proporción de usuarios de

teléfono celular en el ámbito urbano es $72.4 \%$. Además, en $92.5 \%$ de los hogares hay televisor, de los cuales $96 \%$ tienen señal digital de paga o decodificador, y $4 \%$ no tienen señal digital.

Por otra parte, vale destacar que en el referido Foro Mundial Sobre la Educación celebrado en Incheon (República de Corea) se estableció el Marco de Acción de Educación 


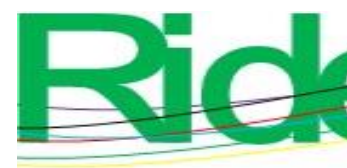

Revista Iberoamericana para la Investigación y el Desarrollo Educativo ISSN $2007-7467$

informal. En pocas palabras, es necesario identificar la importancia de la educación como pieza clave de sensibilización y formación para lograr el desarrollo sostenible, tal como se expuso en la Cumbre de Río en 1992. Aun así, siguen persistiendo diversidad de interpretaciones relacionadas con la educación para el desarrollo sostenible, lo cual repercute en el logro de la transformación del aprendizaje, así como de la evolución de sociedades más justas con nuevos estilos de vida sostenibles.

Por ello, la relevancia de esta investigación radica en generar nuevas líneas de investigación y analizar el significado de los métodos de diseño curricular, pues de ese modo se puede trabajar en innovar e incrementar la calidad de la educación a través de la creación de nuevas herramientas curriculares enfocadas en fortalecer el contenido de los programas de estudios con temas relacionados con el desarrollo sostenible e incorporar estrategias sensoriales didácticas que combinen técnicas con aplicaciones digitales y medios electrónicos. Asimismo, brindar la oportunidad de ampliar el conocimiento derivado de los nuevos retos en la creación, diseño o rediseño curricular de los programas de estudio de las instituciones públicas de educación superior, ya que en la actualidad son incipientes las investigaciones para evaluar la implementación de planes auxiliares que formen parte del método de diseño curricular de la oferta educativa.

\section{Propósito de la investigación}

Analizar el significado de los métodos de diseño curricular e identificar áreas de oportunidad para mitigar los efectos del confinamiento en el plan curricular de la modalidad escolarizada con el fin de impulsar la continuidad de la educación para el desarrollo sostenible a partir de la etapa de retorno a las escuelas con las nuevas formas de interacción en el proceso de enseñanza y aprendizaje. Igualmente, proporcionar una herramienta estratégica de gestión para situaciones críticas derivadas de las incidencias que pudieran detener de forma temporal o permanente el proceso de aprendizaje y crear un plan de diseño curricular auxiliar sostenible que incluya estrategias por medio del aprendizaje híbrido para situaciones críticas de contingencia y contenidos relacionados con la educación para el desarrollo sostenible en todas las áreas del conocimiento. De este modo, se procura coadyuvar en la transformación de las competencias docentes y habilidades de aprendizaje del estudiante de forma resiliente y abierta al cambio, así como en las acciones de la agenda 2030 para la educación del desarrollo sostenible. 


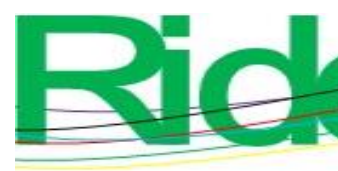

Revista Iberoamericana para la
Investigación y el Desarrollo Educativo ISSN $2007-7467$

\section{Hipótesis}

Se infiere que el método de diseño curricular del modelo escolarizado de las instituciones públicas de educación superior no garantiza que el contenido programático logre alcanzar los objetivos para el desarrollo de competencias y habilidades en los estudiantes en situaciones críticas que propician el cierre de las escuelas, ya que solo se implementa de manera alternativa y provisional el uso de herramientas electrónicas y digitales para dar continuidad al proceso de aprendizaje.

Actualmente, la covid-19 es una pandemia que afecta a la mayor parte de los países en el mundo. El virus ha propiciado el confinamiento de las personas a nivel mundial, lo que ha obligado a buscar otras formas de interacción. Esto ha impactado a todos los sectores, especialmente al educativo, de ahí que se hayan planteado los siguientes cuestionamientos:

¿Cuáles son las innovaciones que favorecerían la educación para el desarrollo sostenible y con ello incrementar la calidad de la educación?

¿Cuál es la importancia de incorporar dentro de la estructura del método de diseño curricular un plan auxiliar sostenible, que incluya estrategias didácticas para las situaciones críticas que impiden dar continuidad al proceso de enseñanza y aprendizaje en el aula?

Con esto se procura comprender la importancia que tiene la realización de un estudio cualitativo para diseñar una herramienta que integre un plan curricular auxiliar sostenible con estrategias, tipos y modelos de aprendizaje para momentos de contingencia derivado de las situaciones críticas que impiden dar continuidad al proceso de enseñanza y aprendizaje en el aula.

Además, se busca incorporar dentro de la estructura del método de diseño curricular un plan auxiliar que incluya contenidos relacionados con la educación para el desarrollo sostenible, así como estrategias didácticas para favorecer la formación tanto de docentes como de estudiantes para la educación del futuro.

\section{Método}

El estudio se basó en los métodos analítico, sistémico e inductivo, a través de los cuales se obtuvieron datos acerca del contexto de las principales teorías de aprendizaje constructivista, métodos de diseño curricular, postulados sobre la educación para el desarrollo sostenible, tendencias tecnológicas en la educación, tipos y modelos de aprendizaje y formas de enseñanza. 


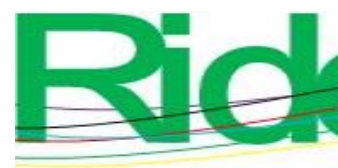

Revista Iberoamericana para la
Investigación y el Desarrollo Educativo
ISSN $2007-7467$

La información se analizó, codificó y categorizó en un software de gestión de bases de datos relacionales. En la herramienta se registraron las características, los componentes, los métodos, las teorías, las tendencias, los tipos, los estilos y los modelos de aprendizaje relacionados con el contexto de la investigación. Luego, con los datos se hizo un comparativo y se definieron las interacciones a través de un diagrama de contexto y relación.

\section{Muestra}

Durante el proceso de investigación cualitativa, primero se hizo un muestreo para tomar una parte representativa de la población, tal es el caso de un método de diseño curricular de tres instituciones públicas de educación superior, tres postulados internacionales para la educación del desarrollo sostenible, dos teorías de aprendizaje constructivista basado en el andamiaje, una teoría para el diseño de estrategias de aprendizaje, así como referencias acerca de los estilos y modelos de aprendizaje.

La población muestra se determinó con base en las características de los recursos en línea ubicados en los sitios web de las instituciones públicas de educación superior, así como de organismos internacionales. A su vez, para calcular la muestra se implementó la técnica no probabilística por conveniencia (McMillan y Schumacher, 2005), al tomar en cuenta que los datos se obtuvieron de documentos primarios disponibles en diversos medios digitales de acceso público, lo cual facilitó el desarrollo de la investigación.

Posteriormente, al proseguir con el análisis, se identificó la ubicación de los documentos primarios; posteriormente se accedió a revisar la información disponible con el fin de seleccionar los datos útiles para la investigación. La población objetivo se delimitó al identificar la población asequible. En consecuencia, para integrar las unidades básicas de análisis, las correcciones realizadas a la muestra inicial se desarrollaron conforme se recopiló y organizó la información.

\section{Instrumentos}

Se utilizó el software de Microsoft Access 2016 para la gestión de bases de datos relacionales. De este modo se logró organizar, ordenar, agrupar y clasificar los datos obtenidos en las fuentes de información primaria de forma sencilla e intuitiva sin necesidad de requerir de un desarrollador. Con dicho programa se puede exportar o convertir la 


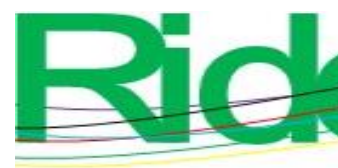

Revista Iberoamericana para la
Investigación y el Desarrollo Educativo
ISSN $2007-7467$

información en diversos formatos electrónicos (PDF, Excel o SQL). Además, permite almacenar, buscar, manejar y compartir datos, así como analizar e imprimir información. La base datos está compuesta por tablas que tienen asignada una clave principal que establece las condiciones de uso para garantizar su validez e integridad referencial en la interacción entre ellas, lo que evita eliminar, modificar o duplicar los datos.

\section{Validez}

Se diseñó una herramienta informática para almacenar la información analizada en los documentos primarios. El diseño de la base de datos tiene como respaldo la validez del constructo en donde cada uno de los campos creados corresponde a los códigos, categorías y subcategorías de las variables de las unidades básicas de análisis, generadas a partir de los métodos de diseño curricular, teorías de andamiaje, postulados internacionales de educación para el desarrollo sostenible, referentes de estrategias, tipos, estilos y modelos de aprendizaje.

\section{Confiabilidad}

La calidad y actualización del contenido de la información de fuentes primarias respalda el prestigio de los datos publicados en los sitios web de las instituciones públicas de educación superior, así como de organismos internacionales.

\section{Objetividad}

El contenido de la base de datos es confiable por su carácter ontológico, ya que los datos se registraron con el lenguaje literal obtenido de las fuentes de información primaria. Es así como las categorías y subcategorías de las variables están respaldadas epistemológicamente, pues se basan en la conceptualización propia de las instituciones educativas del nivel superior, así como de los organismos internacionales. En consecuencia, la investigación tiene un carácter ético, basado en la imparcialidad debido a que la finalidad del estudio se enfoca en aportar conocimiento científico. 


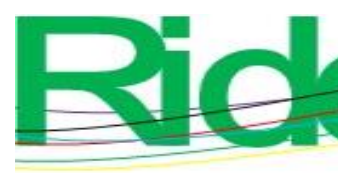

Revista Iberoamericana para la Investigación y el Desarrollo Educativo ISSN $2007-7467$

\section{Procedimiento}

Para recabar información se utilizó el método de recolección de documentos y materiales, así como la técnica de análisis de contenido de documentos, registros, materiales y artefactos, por lo que se omite la participación de encuestadores y la petición de permisos para aplicar algún instrumento debido a que se dispone de una gran diversidad de documentos primarios de acceso público.

Para proseguir con la recolección de datos, se diseñó un proceso de búsqueda de información para organizarla e interpretarla. Este consistió en realizar búsquedas en el acervo de instituciones públicas de educación superior, así como de organismos internacionales relacionados con los programas de la educación para el desarrollo sostenible. También se analizaron las tendencias y factores externos que pudieran influir en el eje de la investigación en diversas fuentes de información primaria.

La información se organizó de forma sistémica, a través de las interacciones entre los códigos de las unidades básicas de análisis, así como de las variables, categorías y subcategorías identificadas en los datos de la población objeto de estudio. Además, al realizar el análisis de información se crearon nuevos campos que no habían sido considerados en la base de datos original.

\section{Plan para la recolección de datos}

Para recolectar los datos se llevó a cabo un plan detallado constituido por procesos que facilitaron la forma de conducir la recolección de la información. Previo al análisis de los datos se adicionaron nuevos planteamientos a la hipótesis: ¿cuál es el método de diseño curricular utilizado en las instituciones públicas de educación superior? ¿Cuáles son sus características? ¿Qué mecanismos incorporan en el método de diseño curricular para situaciones críticas? ¿Cuáles son las innovaciones incorporadas en el método de diseño curricular respecto a las tendencias de la educación a nivel internacional? ¿Cuáles son los postulados de los organismos internacionales relacionados con la educación para el desarrollo sostenible? ¿Cuáles son los postulados que inciden en la educación para el desarrollo sostenible? ¿Cuáles precursores aportan la teoría del andamiaje? ¿Cómo se aplica el andamiaje en el método de diseño curricular? ¿Cuál es el enfoque de cada precursor? ¿Cuál es el impacto de la covid-19 en la educación? ¿Cuál es el papel de las instituciones educativas ante el confinamiento por la covid-19? ¿Cómo se dio la 


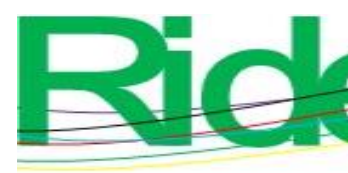
Revista Iberoamericana para la
Investigación y el Desarrollo Educativo
ISSN $2007-7467$

continuidad del proceso de aprendizaje durante el confinamiento? ¿Cuál es la estrategia didáctica aplicada a través de medios digitales y electrónicos? ¿Cuáles son sus características? ¿Cuáles estilos y tipos de aprendizaje utilizan?

Además, durante el proceso de recolección de datos se identificaron las fuentes de información primaria en el acervo de los sitios web de las instituciones públicas de educación superior, así como en los organismos internacionales objeto de estudio. Al realizar las búsquedas se hizo el registro de las direcciones electrónicas en donde se recuperaron los documentos y se almacenaron en una carpeta electrónica para realizar el registro de la información en la base de datos.

Por otra parte, al concluir el registro de la información se utilizaron las herramientas disponibles en el software de gestión de bases de datos para generar informes, los cuales despliegan una matriz que muestra la interacción de las veintidós variables con las ciento treinta y ocho categorías identificadas para ser analizadas e interpretadas. También se utilizó la herramienta de integridad referencial de la base de datos para realizar el mapeo de las unidades básicas de análisis para destacar los datos representativos de la investigación.

En cuanto a la descripción del diseño de la base de datos, primero se crearon veintidós tablas que representan las variables de las unidades básicas de análisis objeto de estudio, las cuales contienen campos que forman parte de las categorías del registro y almacenamiento de la información. Posteriormente, con las tablas se generaron veintidós consultas que representan los códigos de las unidades básicas de análisis; su función radica en recuperar datos específicos de las tablas al realizar búsquedas dentro de la base de datos.

Luego se diseñaron tres formularios que representan el ambiente gráfico de acceso a la base de datos; su interfaz contiene botones de comando para agregar un nuevo registro, eliminarlo, cerrar el formulario, imprimir tabla y salir.

También se crearon tres informes que despliegan la información en forma de matriz con las interacciones correspondientes entre variables y categorías. La información se podrá exportar a otro software procesador de texto, hojas de cálculo o envío de mensajería electrónica. Finalmente, se crearon tres macros para adicionar funciones como generar un formato de contenido portable (PDF) o bien importar la base de datos a un lenguaje de consulta estructurada (SQL).

Respecto a la codificación de los datos, se realizó a través de un proceso sistémico en donde se asignaron códigos tanto a las variables como a las categorías que conforman la 


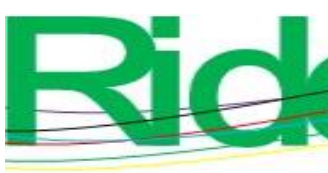

Revista Iberoamericana para la Investigación y el Desarrollo Educativo ISSN 2007 - 7467

base de datos, lo cual facilitó el análisis, la conceptualización de la información, así como la interacción de los datos, como se muestra en la tabla 1.

Tabla1. Codificación de variables y categorías que conforman la base de datos

\begin{tabular}{|c|c|c|}
\hline Código y nombre de la variable & $\begin{array}{c}\mathrm{N} .^{\circ} \\
\text { camp } \\
\text { os }\end{array}$ & Categorías \\
\hline I. Referencia del método de diseño curricular & 3 & $\begin{array}{l}\text { Estructura, lineamientos, } \\
\text { metodología. }\end{array}$ \\
\hline II. Institución & 3 & UNAM, UAM, IPN. \\
\hline III. Vigencia del método curricular & 4 & Actualizado, nuevo, vigente. \\
\hline $\begin{array}{l}\text { IV. Fundamento de la estructura del método } \\
\text { curricular }\end{array}$ & 5 & Propósito, normatividad. \\
\hline V. Estructura del método curricular & 3 & Anteproyecto, propuesta. \\
\hline $\begin{array}{l}\text { VI. Componentes de la estructura del método } \\
\text { curricular }\end{array}$ & 3 & $\begin{array}{l}\text { Análisis de pertinencia, marco } \\
\text { legal. }\end{array}$ \\
\hline VII. Tipo de método curricular & 3 & $\begin{array}{l}\text { Sistémico, modular, marco } \\
\text { normativo. }\end{array}$ \\
\hline $\begin{array}{l}\text { VIII. Características del modelo curricular } \\
\text { sistémico }\end{array}$ & 1 & Basado en procesos. \\
\hline IX. Características del modelo curricular modular & 1 & Estructuras cognitivas. \\
\hline $\begin{array}{l}\text { X. Dimensiones de la educación para el desarrollo } \\
\text { sostenible }\end{array}$ & 4 & $\begin{array}{l}\text { Contenido de aprendizaje, } \\
\text { pedagogía. }\end{array}$ \\
\hline $\begin{array}{l}\text { XI. Temáticas adicionales a incorporar en } \\
\text { contenido }\end{array}$ & 7 & $\begin{array}{l}\text { Cambio climático, consumo y } \\
\text { producción sostenible. }\end{array}$ \\
\hline XII. Pedagogía para el aprendizaje interactivo & 3 & $\begin{array}{l}\text { Aprendizaje orientado hacia la } \\
\text { acción. }\end{array}$ \\
\hline XIII. Contribución de la Unesco & 9 & $\begin{array}{l}\text { Interacción con objetivos de } \\
\text { desarrollo sostenible }\end{array}$ \\
\hline XIV. Interacción de objetivo 4. & 10 & $\begin{array}{l}\text { Objetivos } 1,3,5,8,10,11,12,13, \\
15,16 \text { y } 17\end{array}$ \\
\hline XV. Interacción de objetivos $6,9,11,13$ y 15 & 12 & $\begin{array}{l}\text { Objetivos } 1 \text { al } 5,7,8,10,12,14, \\
16 \text { y } 17\end{array}$ \\
\hline XVI. Interacción de objetivo 14 & 13 & Objetivos 1 al 5, 8 al 13, 15 y 17 \\
\hline XVII. Interacción de objetivos 11 y 16 & 7 & Objetivos 1,3 al 5, 10,13 y 17 \\
\hline XVIII. Interacción de objetivo 11 y 16 & 13 & $\begin{array}{l}\text { Objetivos } 1 \text { al } 6,8,10,12 \text { al } 15 \mathrm{y} \\
17\end{array}$ \\
\hline XIX. Interacción de objetivo 4, 9 y 16 & 8 & $\begin{array}{l}\text { Objetivos } 1,5,6,8,10,11,13 \mathrm{y} \\
17\end{array}$ \\
\hline XX. Tipos de aprendizaje & 20 & Asincrónico, híbrido o b-learning, \\
\hline
\end{tabular}




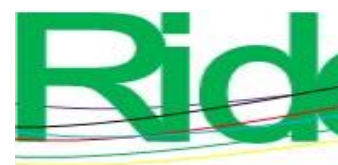

Revista Iberoamericana para la Investigación y el Desarrollo Educativo ISSN $2007-7467$

subcategorías con las cuales se identificaron las interacciones de las variables generadas en las unidades básicas de análisis para examinar los datos obtenidos a través de las fuentes de información descritos con anterioridad.

Por otra parte, la base fundamental del análisis de resultados se basó en el contexto y relación de los datos. La representación gráfica de la figura 2 muestra de forma general la interacción de las unidades básicas de análisis respecto a las categorías y subcategorías de los datos objeto de estudio. En el centro del diagrama se ubica la totalidad del sistema representado por los componentes del contexto de la investigación, los cuales están conectados y diferenciados entre los factores que aportan valor o bien forman parte del flujo de la información por medio de la relación que tienen los datos dentro del sistema, su contexto y su entorno. Es así como las unidades básicas de análisis aportan valor para el análisis de los datos, basados en los objetivos y el planteamiento del problema de investigación. También el método y el proceso de recolección de datos interactúan a través del flujo de la información.

Por otra parte, la representación gráfica de cada componente del diagrama representado de forma específica con el rectángulo ubicado del lado izquierdo contiene los datos que generan insumos, los cuales aportan valor a los componentes del rectángulo del lado derecho que representan los elementos que conforman las categorías de los datos para el análisis de información y también aportan valor de acuerdo con las interacciones que se dan a través del flujo de información utilizada para el análisis de resultados. La representación gráfica central contiene el proceso de investigación en donde se destaca que los objetivos y el planteamiento del problema aportan valor al proceso de análisis de resultados por ser parte del método de investigación cualitativa, así como del proceso de recolección de datos por donde fluye la información. Las flechas continuas representan el aporte de valor en cada uno de los elementos que interactúan en el diagrama y las flechas punteadas simbolizan el flujo de información en cada interacción. 


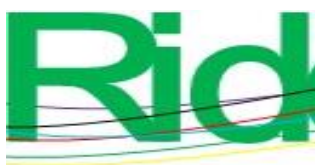

Revista Iberoamericana para la Investigación y el Desarrollo Educativo ISSN 2007 - 7467

Figura 2. Diagrama de contexto y relación de la investigación objeto de estudio

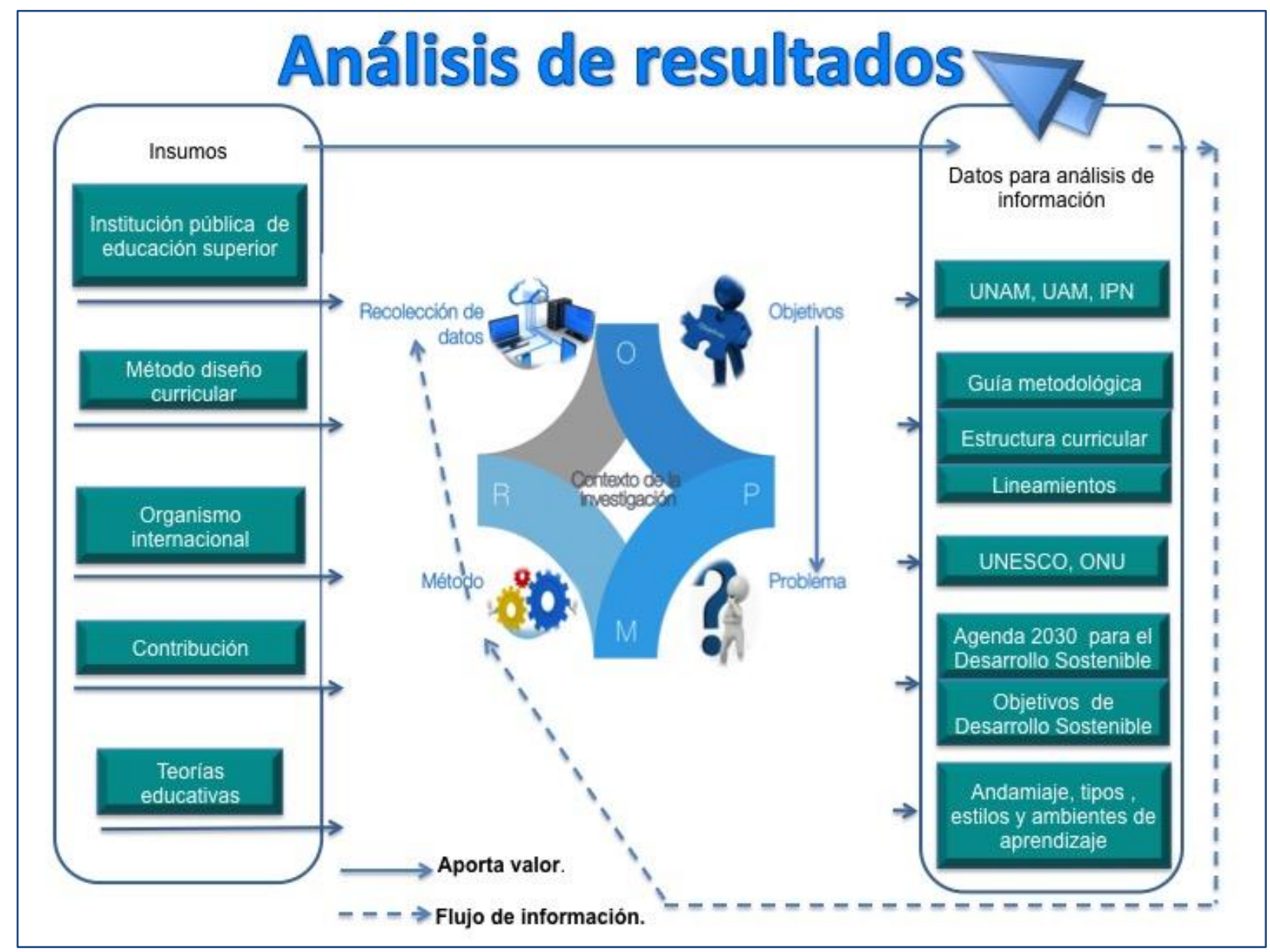

Fuente. Elaboración propia diseñada en Infographics temples for PPT (versión 1.2.3) con imágenes Google

En cuanto al rigor metodológico de los resultados obtenidos en la investigación, se destaca que fueron de carácter ontológico y ético basados en la imparcialidad y objetividad del estudio, como se mencionó con anterioridad.

Por otra parte, durante el análisis de la investigación se abordó la teoría fundamentada (Hernández et al., 2014), con un carácter sistémico debido a que se identificaron diversas categorías al realizar el comparativo de los datos relacionados con el método de diseño curricular, algunas teorías educativas, los objetivos para el desarrollo sostenible, así como la agenda 2030 para el desarrollo sostenible, según métodos y teorías que sustentan cada postulado. También las interacciones se fundamentan en un enfoque de interaccionismo simbólico derivado de la diversidad de interpretaciones identificadas en las fuentes de información consultadas. 


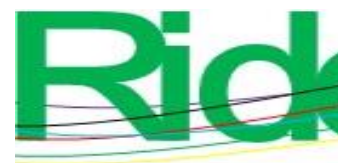

Revista Iberoamericana para la Investigación y el Desarrollo Educativo ISSN 2007-7467

El análisis comenzó a partir de la codificación en la base de datos en donde se identificaron las interacciones. La semántica utilizada tanto unidades básicas de análisis como en las categorías favoreció la interpretación de los datos debido a que se organizaron de manera sistémica. De la figura 3 a la 5 se muestra la interacción de las unidades básicas de análisis respecto a los objetivos, hipótesis y planteamientos formulados.

La primera interacción de datos corresponde a los planteamientos formulados según el método de diseño curricular, sus características y mecanismos empleados para situaciones críticas. Se observó que ninguno de los métodos utilizados por las tres instituciones públicas de educación superior objeto de estudio contempla un plan de contingencia para realizar las adecuaciones pertinentes en caso de alguna situación crítica que impida dar continuidad de forma temporal o permanente al programa de estudios. En cuanto a su estructura, se identificó que el método es diverso, con excepción del modelo curricular, ya que en dos instituciones es sistémico y en una es modular. En el caso de la Universidad Nacional Autónoma de México, su metodología se basa en un anteproyecto que aplica el método de investigación científica (Universidad Nacional Autónoma de México, 2017, pp. 9-11). En el Instituto Politécnico Nacional, el fundamento corresponde a lineamientos institucionales (Instituto Politécnico Nacional, 2012, pp. 3-8) y en la Universidad Autónoma Metropolitana se desconoce el adjetivo que asigna para referir su método debido a que le denomina estructura; sin embargo, toma en cuenta las políticas operacionales, procesos y en el modelo educativo de la institución (Universidad Autónoma Metropolitana, 2005, pp. 63-76), tal como se muestra en la figura 3: 

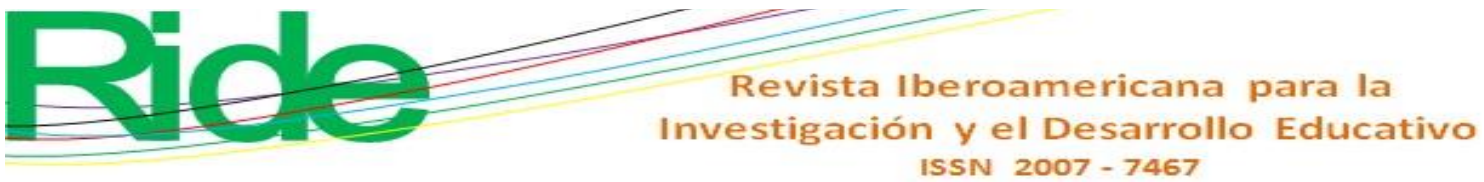

Figura 3. Diagrama de entidad relación respecto a la estructura y fundamento del método de diseño curricular

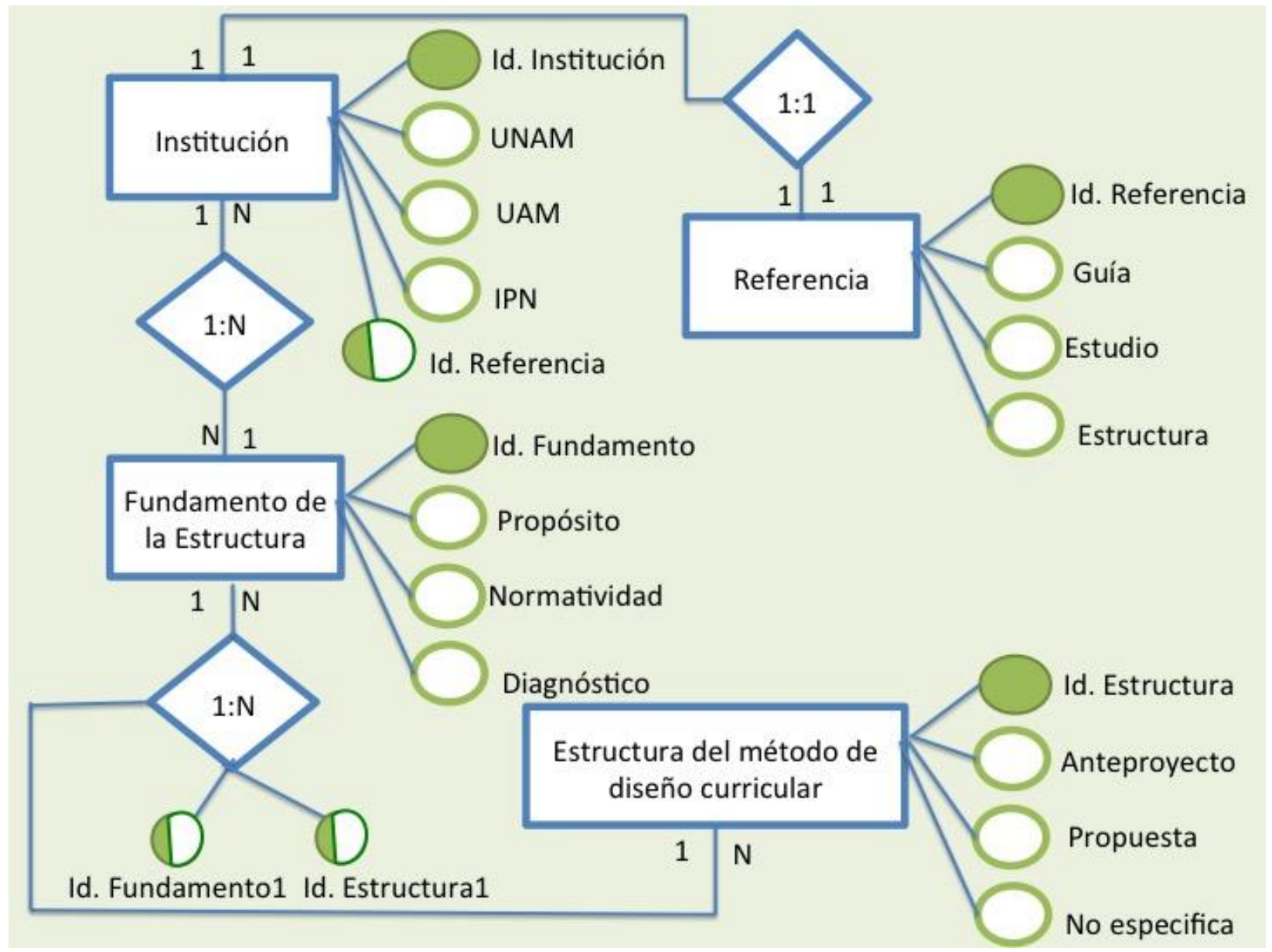

Fuente: Elaboración propia diseñada en Diagrammix (versión 2.16)

Corresponde a la segunda interacción abordar las aportaciones de los organismos internacionales. Se infiere que - debido a la fecha de elaboración del método de diseño curricular en las tres instituciones públicas de educación superior- carecen de apartados específicos para abordar temáticas acerca de la educación para el desarrollo sostenible y de un plan auxiliar para contingencias; no obstante, disponen de programas alternativos para incorporar aspectos relacionados con la sustentabilidad, pero separados de la estructura del diseño curricular. En consecuencia, en la base de datos se generó la interacción con las dimensiones de la agenda 2030 para la educación del desarrollo sostenible de la Unesco, la cual aborda aspectos relacionados con la pedagogía, entornos de aprendizaje, así como temáticas que interactúan con los objetivos de desarrollo sostenible postulados por la ONU, tal como se muestra en la figura 4: 

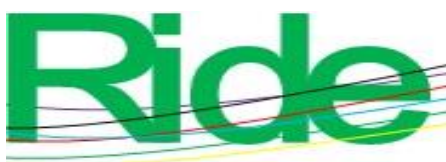

Revista Iberoamericana para la

Investigación y el Desarrollo Educativo

ISSN 2007-7467

Figura 4. Diagrama de entidad relación respecto a las dimensiones y temáticas de la educación para el desarrollo sostenible

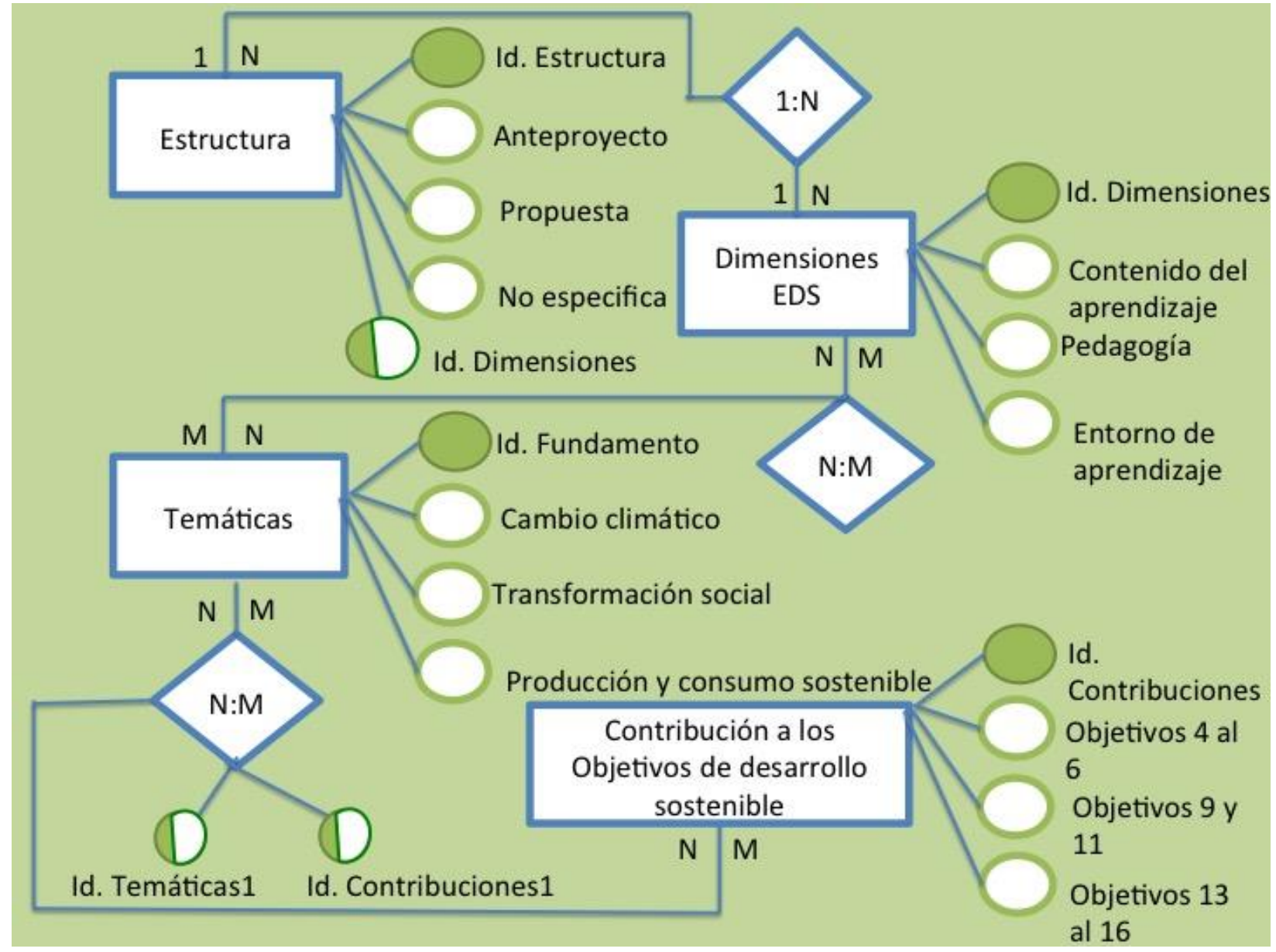

Fuente: Elaboración propia diseñada en Diagrammix (versión 2.16)

Finalmente, la tercera interacción corresponde a las dimensiones de la educación para el desarrollo sostenible respecto a los modelos, estilos y tipos de aprendizaje, los cuales servirán de complemento a considerar en el apartado de modalidad de la propuesta curricular, tal como se muestra en la figura 5: 

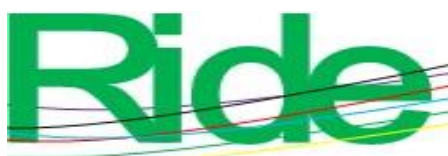

Revista Iberoamericana para la Investigación y el Desarrollo Educativo ISSN 2007 - 7467

Figura 5. Diagrama de entidad relación respecto a la interacción de las dimensiones de la educación del desarrollo sostenible en relación con las modelos, estilos y ambientes de aprendizaje

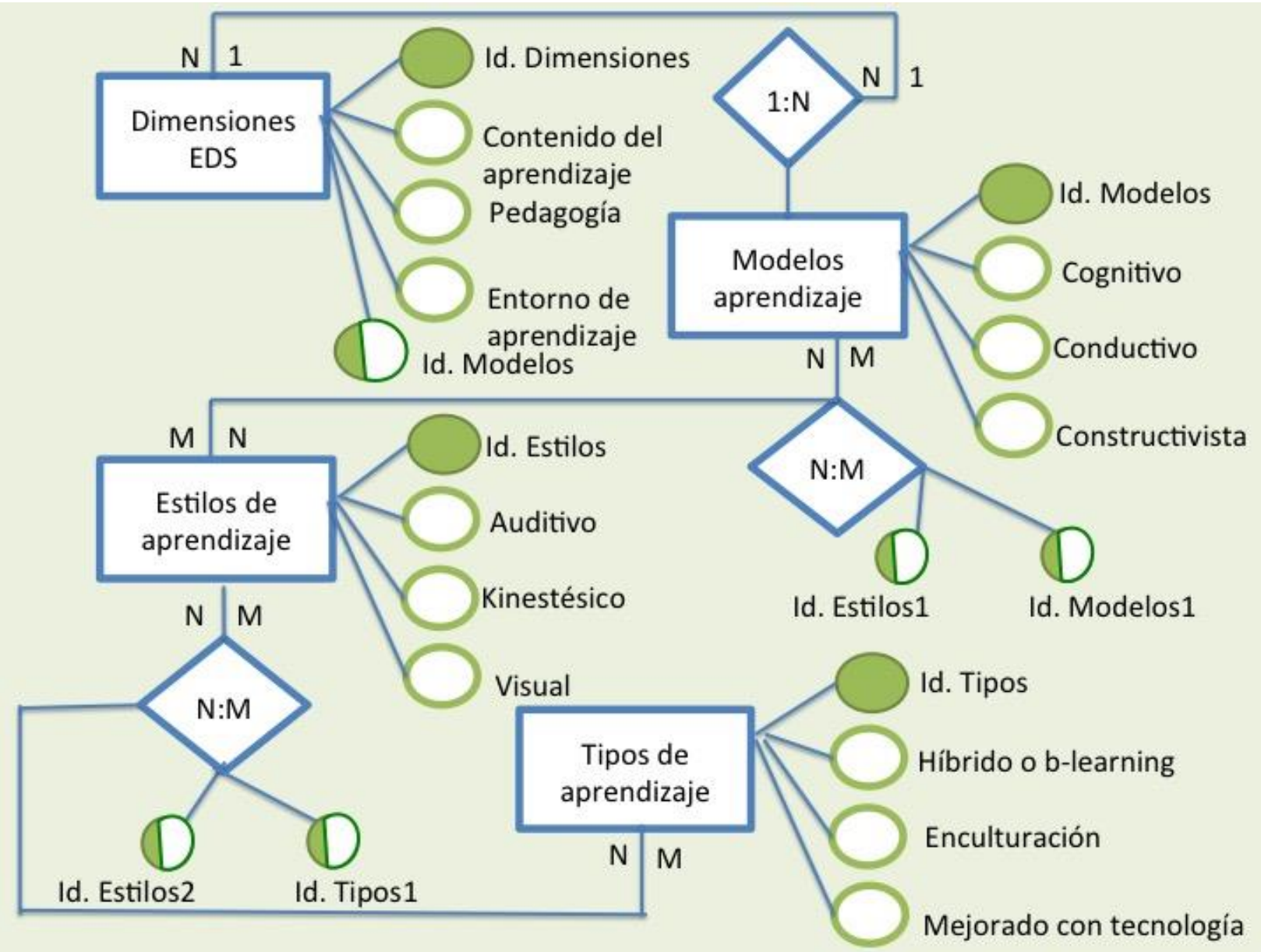

Fuente: Elaboración propia diseñada en Diagrammix (versión 2.16)

Hay que mencionar, además, que como resultado de la investigación se propone una herramienta innovadora de carácter sistémico para apoyar los trabajos realizados en el diseño curricular de las instituciones públicas de educación superior. La herramienta se denomina Modelo conceptual del plan curricular auxiliar sostenible en periodos de contingencia para las instituciones públicas de educación superior. La estructura del modelo está constituida por la interacción de cinco fases, como se aprecia en la figura 6: 


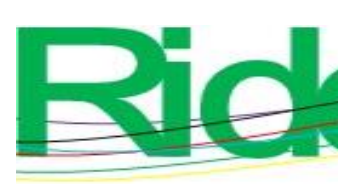

Revista Iberoamericana para la Investigación y el Desarrollo Educativo ISSN 2007 - 7467

- Actividades de formación docente para fortalecer el uso de métodos y herramientas del aprendizaje híbrido o b-learning.

- Estrategias didácticas para el diseño de contenido de educación para el desarrollo sostenible en un modelo híbrido.

Por otra parte, Reimers y Schleicher (2020) presentaron un reporte realizado por la Iniciativa de Innovación Educativa Global de la Escuela de Graduados de Educación de Harvard en colaboración con la Dirección de Educación y Habilidades de la Organización para la Cooperación y el Desarrollo Económicos (OCDE). El informe tiene el propósito de apoyar la decisión de los líderes de los sistemas educativos para implementar planes con modalidades alternativas derivadas del aislamiento social por la pandemia del covid-19, "basado en el análisis de datos de la más reciente encuesta aplicada con PISA” que evalúa los desafíos educativos creados en este contexto. Al respecto, se puntualizan aspectos para evitar la pérdida de aprendizaje, así como priorizar los objetivos curriculares para definir lo que debe aprenderse durante el confinamiento. También comparten un "plan flexible para la continuidad de la instrucción" en donde se formulan dos escenarios para la enseñanza, por medio del modelo híbrido o mixto. La propuesta considera dar clase presencial y en línea a un número específico de estudiantes en cada modalidad. Para ello, el docente deberá planificar previamente las clases, los materiales, así como las estrategias didácticas y las evaluaciones, además de coordinar las clases en ambas modalidades.

También refieren que en el Programa de Evaluación Internacional de Estudiantes (PISA) 2018 de la OCDE muestra que en promedio "el 9\% de los estudiantes de 15 años ni siquiera tiene un lugar tranquilo para estudiar en sus casas. El aprendizaje en línea no solo requiere un lugar para estudiar, sino también una computadora que los estudiantes puedan usar para trabajar en sus casas" (p. 15).

Asimismo, Sanz et al. (2020) advierten en el "informe de los efectos del coronavirus en la educación", lo que forma parte de la iniciativa de la Organización de Estados Iberoamericanos para la Educación, la Ciencia y la Cultura (OEI) en cuanto al rol fundamental de los padres para apoyar a los estudiantes en casa, lo cual también dependerá de la calidad de la plataforma digital. Estos autores puntualizan que la clave de éxito será "la formación del profesorado en el uso de metodologías online y su interacción en el aprendizaje" (p. 10). 


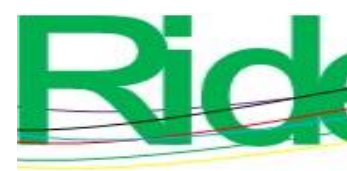

Revista Iberoamericana para la Investigación y el Desarrollo Educativo ISSN $2007-7467$

El documento también señala el impacto del cierre de los centros de aprendizaje respecto a los ajustes que podrían realizarse en los contenidos del currículo, lo cual generaría un incremento en la brecha del conocimiento.

Los primeros dos planteamientos de la hipótesis permitieron estructurar la base de datos referencial. Al continuar con el proceso de investigación en la fase de recolección de datos se formularon nuevos planteamientos con los hallazgos encontrados, para lo cual se agruparon para dar respuesta a los aspectos relacionados con el método de diseño curricular, postulados acerca de la educación para el desarrollo sostenible, situaciones críticas, así como estrategias de aprendizaje, lo cual facilitó el diseño del modelo propuesto.

Finalmente, algo que pudiera limitar la aplicación o implementación de los instrumentos generados en la investigación sería la diversidad de percepciones e interpretaciones de los postulados incorporados en la investigación.

\section{Conclusión}

Tomando como base los resultados de la investigación, se concluye que las instituciones públicas de educación superior deben innovar de forma permanente en cuanto al método de diseño curricular e incorporar herramientas auxiliares en situaciones críticas, que sirvan de complemento al trabajo realizado en la actualización o rediseño curricular con el fin de dar continuidad al proceso de enseñanza y aprendizaje de forma sostenible.

Asimismo, incorporar temas relacionados con la agenda 2030 para el desarrollo sostenible en un plan curricular auxiliar sostenible de forma multidisciplinaria favorece la calidad de la formación de los estudiantes, así como el aprendizaje significativo al aplicar el conocimiento en la prevención de situaciones críticas derivadas de fenómenos naturales ocasionados por el cambio climático, lo cual promueve la prosperidad y el bienestar de la sociedad en general, así como la protección del medio ambiente.

Por otra parte, hasta el momento la hipótesis planteada respecto al "método de diseño curricular no garantiza que el contenido programático logre alcanzar los objetivos para el desarrollo de competencias y habilidades de los estudiantes". No se ha logrado confirmar o rechazar debido a que las instituciones públicas de educación superior objeto de estudio se encuentran en el proceso de implementación de la evaluación y diagnóstico de los saberes adquiridos a través de las estrategias didácticas y herramientas tecnológicas utilizadas durante el proceso de confinamiento derivado de la pandemia de la covid-19. 


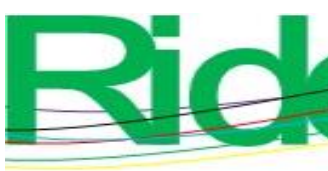

Revista Iberoamericana para la
Investigación y el Desarrollo Educativo ISSN $2007-7467$

\section{Referencias}

Bartolomé, A. y Aiello, M. (2006). Nuevas tecnologías y necesidades formativas: Blended learning y nuevos perfiles en comunicación audiovisual. Telos, 67, 59-67. Recuperado de https://www.researchgate.net/publication/28109534_Nuevas_tecnologias_y_necesid ades_formativas_Blended_Learning_y_nuevos_perfiles_en_comunicacion_audiovis ual

Hernández, R., Fernández, C. y Baptista, L. (2014). Metodología de la investigación. México: McGraw Hill. Recuperado de https://www.uca.ac.cr/wpcontent/uploads/2017/10/Investigacion.pdf

Instituto Nacional de Estadística y Geografía [Inegi] (2019). Encuesta nacional sobre disponibilidad y uso de tecnologías de la información en los hogares ENDUTIH 2019.

Recuperado

de https://www.inegi.org.mx/contenidos/saladeprensa/aproposito/2019/internet2019_N al.pdf

Instituto Politécnico Nacional (2012). Lineamientos para la aprobación de programas académicos en el Instituto Politécnico Nacional. Gaceta politécnica [edición extraordinaria], 15(979). Recuperado de https://www.ipn.mx/assets/files/normatividad/docs/Lineamientos//GAC_970EXT.p df

McMillan, J. y Shchumacher, S. (2005). Investigación educativa (5. a ed.). Madrid: Pearson. Recuperado de https://desfor.infd.edu.ar/sitio/upload/McMillan_J._H._Schumacher_S._2005._Investigacion _educativa_5_ed..pdf

Organización de las Naciones Unidas [ONU] (2015). Agenda 2030. Una agenda universal, transformativa e integrada que anuncia un hito histórico para nuestro mundo. ONU, México. Recuperado de https://www.onu.org.mx/agenda-2030/

Organización de las Naciones Unidas para la Educación, la Ciencia y la Cultura [Unesco] (2012). Educación para el desarrollo sostenible. Recuperado de http://www.lacult.unesco.org/docc/2012_Educ_para_des_sost.pdf

Organización de las Naciones Unidas para la Educación, la Ciencia y la Cultura [Unesco] (2014). Hoja de ruta para la ejecución del programa de acción mundial de 

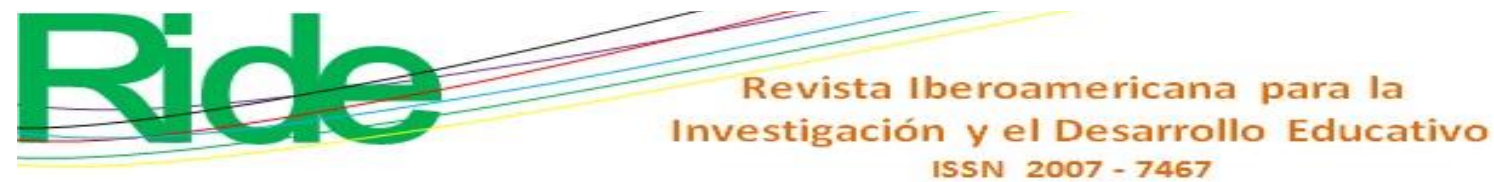

Educación para el Desarrollo Sostenible. Unesco, Francia. Recuperado de https://unesdoc.unesco.org/ark:/48223/pf0000230514_spa

Organización de las Naciones Unidas para la Educación, la Ciencia y la Cultura [Unesco] (2017). La Unesco avanza. La agenda 2030 para el desarrollo sostenible. Organización de las Naciones Unidas para la Educación, la Ciencia y la Cultura, Oficina de Planeación estratégica, Sección de Cooperación con el Sistema de Naciones Unidas. Recuperado de http://www.unesco.org/new/fileadmin/MULTIMEDIA/FIELD/Hanoi/2030_Brochur e_SP.pdf

Organización Mundial de la Salud [OMS] (2020). Fortalecimiento de la preparación para la COVID-19 en las ciudades y otros entornos urbanos. OMS. Recuperado de https://apps.who.int/iris/bitstream/handle/10665/332039/WHO-2019-nCoV-

Urban_preparedness-2020.1-spa.pdf?sequence $=1$ \&isAllowed=

Reimers, F. y Schleicher, A. (2020). Un marco para guiar una respuesta educativa a la pandemia del 2020 del COVID-19. Enseña Perú. Recuperado de https://globaled.gse.harvard.edu/files/geii/files/un_marco_para_guiar_una_respuesta _educativa_a_la_pandemia_del_2020_del_covid-19_.pdf

Salinas, J., De Benito, Pérez, A. y Gisbert, M. (2018). Blended learning, más allá de la clase presencial. RIED, Revista Iberoamericana de Educación a Distancia, 21(1), 195-213.

Recuperado de http://revistas.uned.es/index.php/ried/article/view/18859/16909

Sanz et al. (2020). Efectos de la crisis del coronavirus en la educación. Recuperado de https://www.oei.es/Educacion/Noticia/oei-analiza-como-afectara-el-cierre-colegioscoronavirus

Universidad Autónoma Metropolitana (2005) Estructura curricular. Unidad Cuajimalpa $\left(2 .^{a} \quad\right.$ ed.) Recuperado de http://www.cua.uam.mx/pdfs/conoce/estructura_currucular/051201.Estructura_curri cular.pdf

Universidad Nacional Autónoma de México (2017). Guía metodológica para elaborar la fundamentación del plan de estudios. Coordinación de Desarrollo Educativo e Innovación Curricular. Recuperado de https://www.codeic.unam.mx/wpcontent/uploads/2016/08/documentosCODEIC/Guia_fundamentacion_PE2.pdf 